\title{
Rural-urban difference in colorectal cancer mortality
}

\author{
Tomoya Urakawa, Akihiro Saitsu, Jun Watanabe, Kazuhiko Kotani ${ }^{凶}$ \\ Division of Community and Family Medicine, Center for Community Medicine, Jichi Medical University, Shimotsuke-City, Tochigi, Japan. \\ $\square$ Corresponding author: Kazuhiko Kotani, M.D., Ph.D., Division of Community and Family Medicine, Center for Community Medicine, Jichi Medical \\ University, 3311-1 Yakushiji, Shimotsuke-City, Tochigi 329-0498, Japan. Tel.: +81-285-58-7394; Fax: +81-285-44-0628; E-mail: kazukotani@jichi.ac.jp. \\ () The author(s). This is an open access article distributed under the terms of the Creative Commons Attribution License (https://creativecommons.org/licenses/by/4.0/). \\ See http://ivyspring.com/terms for full terms and conditions.
}

Received: 2021.02.15; Accepted: 2021.03.10; Published: 2021.04.13

Related article: J Cancer 2021; 12(2):474-481. doi:10.7150/jca.49676.

We read with great interest the recent study by Daniel et al., which showed a different response to knowledge and practice in relation to colorectal cancer screening between minority and majority populations in the deep south of the U.S. [1]. As Daniel et al. stated [1], rurality is a possible contributor to increased mortality in patients with colorectal cancer because several factors related to lifestyle, and socioeconomics and healthcare systems are associated with the diagnosis and clinical course of this disease. Thus, the study assumed the difference in responses to colorectal cancer screening between rural and urban residents - interestingly, however, few differences were found [1]. This might be an unexpected finding, as it is generally considered that rural-urban health inequity in cancer treatment is an issue that remains to be resolved.

Actually, what is the worldwide status of the rural-urban difference in colorectal cancer mortality, which is an ultimate outcome of screening? We performed a search of the PubMed database to identify original articles published prior to December 2020, with a population-based cohort design that reported the colorectal cancer mortality between rural and urban areas. The keywords were "rural[All Fields] OR urban[All Fields]) AND ("rectal neoplasms"[MeSH Terms] OR ("rectal"[All Fields]
AND "neoplasms"[All Fields]) OR "rectal neoplasms"[All Fields] OR ("rectum"[All Fields] AND "cancer"[All Fields]) OR "rectum cancer"[All Fields]) AND ("mortality"[Subheading] OR "mortality" [All Fields] OR "mortality" [MeSH Terms]) AND English[lang]. We obtained the hazard ratio or relative risk for cancer death between rural and urban areas, with adjustment for various co-variates.

In total, 143 English language articles were identified using the keywords. After evaluating the full text, five articles were finally eligible for the present review (Table 1) [2-6]. As a result, one study reported that the mortality risk was high in urban areas [2], while two studies reported that the mortality risk was high in rural areas $[3,5]$. The remaining two studies reported no clear difference in risk between rural and urban areas [4, 6].

The results of the present review were therefore controversial; namely, a rural-urban difference in colorectal cancer mortality was not obviously proven. Of note, the present findings appear to be in line with the results reported by Daniel et al. [1]. While Daniel et al. hypothesized that rural residents often acknowledge a family history of cancer and cope with it well, more studies are warranted to clarify the detailed reasons for these findings.

Table 1. The hazard ratio or relative risk of colorectal cancer deaths between rural and urban areas

\begin{tabular}{|c|c|c|c|c|c|c|c|}
\hline $\begin{array}{l}\text { Study/Country } \\
\text { [reference No.] }\end{array}$ & $\begin{array}{l}\text { Cohort } \\
\text { duration }\end{array}$ & $\begin{array}{l}\text { Population } \\
\text { (number) }\end{array}$ & Age (number) & Cancer type & Residence & $\begin{array}{l}\text { Hazard ratio/relative risk } \\
\text { (95\% confidence interval) }\end{array}$ & Adjusted co-variables \\
\hline \multirow[t]{5}{*}{$\begin{array}{l}\text { Kassim, 2019/ } \\
\text { China [6] }\end{array}$} & $2007-2011$ & $\begin{array}{l}\text { Male } 143 \\
\text { Female } 91\end{array}$ & 54.96 years & Right colon & $\begin{array}{l}\text { Urban } \\
\text { Rural }\end{array}$ & $\begin{array}{l}1.0 \text { (reference) } \\
1.082(0.765-1.529)\end{array}$ & \multirow[t]{5}{*}{$\begin{array}{l}\text { Age, gender, smoking, drinking, residence, } \\
\text { cancer grade, cancer stage, chemotherapy }\end{array}$} \\
\hline & & Male 147 & 53.93 years & Left colon & Urban & 1.0 (reference) & \\
\hline & & Female 94 & & & Rural & $1.151(0.666-1.988)$ & \\
\hline & & Male 369 & 56.86 years & Rectum & Urban & 1.0 (reference) & \\
\hline & & Female 234 & & & Rural & $0.934(0.752-1.159)$ & \\
\hline
\end{tabular}




\begin{tabular}{|c|c|c|c|c|c|c|c|}
\hline $\begin{array}{l}\text { Study/Country } \\
\text { [reference No.] }\end{array}$ & $\begin{array}{l}\text { Cohort } \\
\text { duration }\end{array}$ & $\begin{array}{l}\text { Population } \\
\text { (number) }\end{array}$ & Age (number) & Cancer type & Residence & $\begin{array}{l}\text { Hazard ratio/relative risk } \\
\text { ( } 95 \% \text { confidence interval) }\end{array}$ & Adjusted co-variables \\
\hline $\begin{array}{l}\text { Feller, 2018/ } \\
\text { Swiss [5] }\end{array}$ & $2000-2008$ & $\begin{array}{l}\text { Male 5,700 } \\
\text { Female } 4,388\end{array}$ & $\begin{array}{l}<50 \text { years }(668) \\
50-64 \text { years }(3,007) \\
65-74 \text { years }(3,180) \\
75-84 \text { years }(3,233)\end{array}$ & Colorectum & $\begin{array}{l}\text { Urban } \\
\text { Rural }\end{array}$ & $\begin{array}{l}1.0 \text { (reference) } \\
1.15(1.02-1.30)\end{array}$ & $\begin{array}{l}\text { Age, civil status, nationality, urbanity, } \\
\text { residence, cancer localization, cancer stage, } \\
\text { socioeconomic position }\end{array}$ \\
\hline $\begin{array}{l}\text { Hines, 2014/ } \\
\text { United States [4] }\end{array}$ & 2000-2012 & $\begin{array}{l}\text { Male } 10,702 \\
\text { Female } 9,742\end{array}$ & $\begin{array}{l}45-64 \text { years }(9,675) \\
65-74 \text { years }(5,890) \\
75-85 \text { years }(4,879)\end{array}$ & Colorectum & $\begin{array}{l}\text { Urban } \\
\text { Rural }\end{array}$ & $\begin{array}{l}1.0 \text { (reference) } \\
1.02(0.94-1.12)\end{array}$ & $\begin{array}{l}\text { Age, gender, race, cancer stage, cancer } \\
\text { grade, geography, treatment (surgery, } \\
\text { chemotherapy or radiation), socioeconomic } \\
\text { status }\end{array}$ \\
\hline $\begin{array}{l}\text { Hines, 2012/ } \\
\text { United States [3] }\end{array}$ & $1992-2007$ & $\begin{array}{l}\text { Male 7,365 } \\
\text { Female 7,809 }\end{array}$ & $\begin{array}{l}\text { Rural } 68.4 \text { years } \\
\text { Urban } 65.8 \text { years }\end{array}$ & $\begin{array}{l}\text { Colon } \\
\text { Rectum }\end{array}$ & $\begin{array}{l}\text { Urban } \\
\text { Rural } \\
\text { Urban } \\
\text { Rural }\end{array}$ & $\begin{array}{l}1.0 \text { (reference) } \\
1.15(1.01-1.32) \\
1.0 \text { (reference) } \\
1.13(0.91-1.41)\end{array}$ & $\begin{array}{l}\text { Age, gender, cancer stage, cancer grade, } \\
\text { treatment (surgery or radiation) }\end{array}$ \\
\hline \multirow[t]{2}{*}{$\begin{array}{l}\text { Vassallo, 1994/ } \\
\text { Uruguay [2] }\end{array}$} & 1988-1992 & $\begin{array}{l}\text { Male } 1,121,250 \\
\text { Female } 1,209,700\end{array}$ & $\begin{array}{l}<55 \text { years }(1,664,150) \\
55-64 \text { years }(316,450) \\
65-74 \text { years }(209,500) \\
>75 \text { years }(140,850)\end{array}$ & Colon & $\begin{array}{l}\text { Urban } \\
\text { Rural }\end{array}$ & $\begin{array}{l}\text { Male } 1.50(1.32-1.70) \\
\text { Female } 1.17(0.34-1.34) \\
\text { Male } 1.0 \text { (reference) } \\
\text { Female } 1.0 \text { (reference) }\end{array}$ & Age, residence \\
\hline & & & & Rectum & $\begin{array}{l}\text { Urban } \\
\text { Rural }\end{array}$ & $\begin{array}{l}\text { Male } 1.89(1.51-2.35) \\
\text { Female } 1.35 \text { (1.06-1.72) } \\
\text { Male } 1.0 \text { (reference) } \\
\text { Female } 1.0 \text { (reference) }\end{array}$ & \\
\hline
\end{tabular}

\section{References}

1. Daniel C, Aly S, Bae S, et al. Differences related to cancer screening by minority and rural/urban status in the Deep South: population-based survey results. J Cancer. 2021; 12: 474-81.

2. Vassallo A, de Stéfani E, Ronco A, et al. Urbanization gradients and cancer mortality in Uruguay, 1988-1992. Int J Cancer. 1994; 59: 345-50.

3. Hines RB, Markossian TW. Differences in late-stage diagnosis, treatment, and colorectal cancer-related death between rural and urban African Americans and whites in Georgia. J Rural Health. 2012; 28: 296-305.

4. Hines R, Markossian T, Johnson A, et al. Geographic residency status and census tract socioeconomic status as determinants of colorectal cancer outcomes. Am J Public Health. 2014; 104: e63-71.

5. Feller A, Schmidlin K, Bordoni A, et al. Socioeconomic and demographic inequalities in stage at diagnosis and survival among colorectal cancer patients: evidence from a Swiss population-based study. Cancer Med. 2018; 7: 1498-510.

6. Abasse Kassim S, Tang W, Abbas M, et al. Clinicopathologic and epidemiological characteristics of prognostic factors in post-surgical survival of colorectal cancer patients in Jiangsu Province, China. Cancer Epidemiol. 2019; 62: 101565 . 